IZA DP No. 5782

\title{
Symmetric and Asymmetric Committees
}

Ruth Ben-Yashar

Leif Danziger

June 2011 


\title{
Symmetric and Asymmetric Committees
}

\author{
Ruth Ben-Yashar
}

Bar-Ilan University

Leif Danziger

Ben-Gurion University

and IZA

\section{Discussion Paper No. 5782 \\ June 2011}

IZA

P.O. Box 7240

53072 Bonn

Germany

Phone: +49-228-3894-0

Fax: +49-228-3894-180

E-mail: iza@iza.org

Any opinions expressed here are those of the author(s) and not those of IZA. Research published in this series may include views on policy, but the institute itself takes no institutional policy positions.

The Institute for the Study of Labor (IZA) in Bonn is a local and virtual international research center and a place of communication between science, politics and business. IZA is an independent nonprofit organization supported by Deutsche Post Foundation. The center is associated with the University of Bonn and offers a stimulating research environment through its international network, workshops and conferences, data service, project support, research visits and doctoral program. IZA engages in (i) original and internationally competitive research in all fields of labor economics, (ii) development of policy concepts, and (iii) dissemination of research results and concepts to the interested public.

IZA Discussion Papers often represent preliminary work and are circulated to encourage discussion. Citation of such a paper should account for its provisional character. A revised version may be available directly from the author. 
IZA Discussion Paper No. 5782

June 2011

\section{ABSTRACT}

\section{Symmetric and Asymmetric Committees}

This paper studies the assignment of decision makers to two committees that make decisions by a simple majority rule. There is an even number of decision makers at each of various skill levels and each committee has an odd number of members. Surprisingly, even with the symmetric assumptions in the spirit of Condorcet, a symmetric composition of committees is not always optimal. In other words, decision makers with different skill levels should not generally be evenly divided among the committees. However, in the special case of only two skill levels, it is optimal to compose the committees evenly.

JEL Classification: D70, D71, D72

Keywords: committees, collective decision making, simple majority rule

Corresponding author:

Leif Danziger

Department of Economics

Ben-Gurion University

Beer-Sheva 84105

Israel

E-mail: danziger@bgu.ac.il 


\section{Introduction}

Committee decision making under uncertainty commonly occurs in such fields as management, law, and medicine where organizations need to make decisions on whether to accept or reject various proposals. Typical examples include a firm that has to decide whether an investment project should be carried out or not, a university that must make an acceptance or rejection decision for each applicant, and a court system that has to determine the guilt or innocence of each defendant. To decide which of two possible states of the world occurs, an organization may employ decision makers who will strive to make the optimal decision for the organization on each proposal. ${ }^{1}$ Due to private information, however, the decision makers may have different judgements about the true state of the world. ${ }^{2}$ Presumably, therefore, a committee of decision makers that aggregates their individual assessments is more likely to make the correct decision than would any single individual. ${ }^{3}$

There are many reasons why an organization may need to appoint different committees that work in parallel and independently decide on each of the proposals assigned to it (e.g., the time involved in examining the merits of each proposal or the geographic dispersion of the relevant activities). It is therefore surprising that the extensive literature on committee decision making always assumes a given composition of committees and never considers the question of how the decision makers are allocated to the committees.

An important component of our model is that the decision makers may differ in their

1 Dewatripont and Tirole (1999) assume that the interests of the decision makers do not necessarily coincide with those of the organization and study how to assign committee members who advocate special interests.

2 See, for example, Sah and Stiglitz (1985) and Sah (1991).

3 The idea that "two good men are better than one" can be traced back to Aristotle (350 BCE). The English proverb "two heads are better than one" is first recorded in Heywood (1546). A formal analysis of the aggregational aspects of committee decision making goes back to Condorcet (1785). His approach has been further developed by, among others, Grofman and Feld (1988), Young (1988), and Ladha (1995). On the other hand, Feddersen and Pesendorfer (1998) show that strategic voting considerations may reduce the probability that the unanimity rule leads to correct decisions. See also Campbell (1999) and Palfrey and Rosenthal $(1983,1985)$ who show that under certain circumstances a majority may be less likely to win than a minority. 
ability to identify the true state of the world, which makes the assignment of decision makers into the different committees a nontrivial problem. The committees work in parallel and the organization divides the decision makers between the committees in order to maximize the average probability (over committees and proposals) of making correct decisions. ${ }^{4}$ We focus on the case in which there is symmetry both between the committees and between the alternatives. The symmetry between the committees means that the two committees have the same size and decide on the same number of independent proposals, while the symmetry between the alternatives means that the prior probability of each state is a half for every proposal, that each decision maker's ability to identify the correct state of the world is the same for both states, and that the net benefit to the organization from making a correct decision is the same for each proposal and state. Ours is a common value model where the decision makers share the organization's objective but have private information. Each committee member can vote either for or against a proposal, ${ }^{5}$ and the committees use a simple majority rule to make the collective decision. With our assumptions, informative voting (meaning that committee members vote according to their true assessment) constitutes a Nash equilibrium behavior. ${ }^{6}$ Thus, we assume that committee members vote informatively.

Assuming that there is an even number of decision makers at each skill level, any combination of committees can be obtained from symmetric committees by an exchange of decision members between the committees. In Theorem 1 we show that starting from symmetric com-

\footnotetext{
${ }^{4}$ Early studies of optimal committee decision rules with binary alternatives include Grofman et al. (1983), Nitzan and Paroush (1982), and Shapley and Grofman (1984). More general models include Ben-Yashar and Nitzan (1997) and Ben-Yashar and Kraus (2002). These papers all assume that there is a single committee and that the objective is to maximize the average probability that the committee makes the correct decision. See also Baharad and Nitzan (2007).

5 We do not consider abstentions. Presumably, the organization would not employ decision makers with so little ability that it would be optimal for them not to participate in the decision making, i.e., abstain. Börgers (2004) presents a symmetric private value model in which abstentions are optimal due to a cost of voting.

6 The reason is that the probability that any particular committee member is pivotal is the same in situations where the correct decision is to accept the proposal as in situations where the correct decision is to reject it. See Austen-Smith and Banks (1996) and Ben-Yashar and Milchtaich (2007).
} 
mittees, an exchange of decision makers with one skill level in one committee for the same number of decision makers with another skill level from the other committee reduces the average probability of making correct decisions. However, in Theorem 2 we show that starting from symmetric committees, an exchange of decision makers with different skill levels in one committee with decision makers with other skill levels from the other committee may increase the average probability of making correct decisions.

The intuition of Theorem 1 is that the more higher skilled committee members there are, the less likely it is that a particular higher skilled member is pivotal. Therefore, the probability that a simple majority makes the correct decision increases at a decreasing rate with the number of decision makers of the same higher skill level that are replacing decision makers with the same lower skill level. As a consequence, in the special case of only two types of decision makers, symmetric committees are optimal.

The surprising result in Theorem 2 is due to the interaction of decision makers with more than two skill levels. The effect on the average probability that a committee makes the correct decision if some of the decision makers that have different skill levels are replaced by the same number of decision makers that have other skill levels is ambiguous and depends on the skill levels of both the replaced decision makers and the decision makers remaining in the committee. Accordingly, even with the symmetry assumptions in the spirit of Condorcet, a symmetric composition of committees is generally not optimal. That is, decision makers with different skill levels should not generally be evenly divided among the committees.

To illustrate that asymmetric committees may be optimal, suppose that the organization employs ten decision makers with two decision makers at each of five different skill levels as defined by the probabilities of making the correct assessment. Suppose also that the probabilities of making the correct assessment of a proposal are $0.9,0.75,0.74,0.72$, and 0.6 , respectively, for the five skill levels. We now compare the asymmetric case in which one committee has decision makers with the probabilities $0.9,0.9,0.74,0.6$, and 0.6 of making the correct assessment, and the other committee has decision makers with the probabilities 
$0.75,0.75,0.74,0.72$, and 0.72 of making the correct assessment with the symmetric case in which the decision makers are evenly distributed among the two committees (i.e., in both committees there is one decision maker at each level of probability of making the correct assessment). As we will see, the average probability of making the correct decisions is higher for asymmetric committees than for symmetric committees.

In this example, the asymmetric committees are obtained from symmetric committees by exchanging a pair of decision makers with skill levels 0.9 and 0.6 with a pair of decision makers with skill levels 0.75 and 0.72 between the two committees. Since $0.9 * 0.6=0.75 * 0.72$, the probability that the committees make the correct decision are unaffected by the case in which all the exchanged decision makers make the correct assessment. Hence, the exchange of decision makers can be of benefit only in the case that exactly one of the exchanged decision makers in each pair makes the correct assessment. Now, the difference in the probability that exactly one decision maker in a pair with skill levels 0.9 and 0.6 and the probability that exactly one decision maker in a pair with skill levels 0.75 and 0.72 make the correct decision is $0.9+0.6-0.75-0.72$. In order to make a difference it is also required that exactly two of the three remaining decision makers with skill levels $0.9,0.6$, and 0.74 in one committee, and exactly two of the three remaining decision makers with skill levels $0.75,0.72$, and 0.74 in the other committee make the correct assessment. The latter requires that exactly one of the decision makers with skill levels 0.9 and 0.6 as well as one of the decision makers with skill levels 0.75 and 0.72 make the correct assessment. It follows that the difference in the probabilities that the asymmetric committees and the symmetric committees make the correct decisions is $\frac{1}{2}(0.9+0.6-0.75-0.72)^{2} * 0.74=3.33 \times 10^{-4}$. Consequently, it is not optimal to divide the decision makers evenly between the committees.

\section{The Model}

We consider an organization that needs to make binary decisions on whether to accept or reject each of various independent proposals. The correct decision for a particular proposal 
depends on which of two states of the world is relevant for this proposal, but the actual state is unknown to the organization at the time the decision must be made. The prior probability of each state of the world is half for each proposal. The net benefit to the organization from each correct decision is identical for all proposals.

Due to the large number of proposals or coordination problems, the organization divides the proposals evenly among two independent committees. Each committee consists of an odd number $N \geq 3$ of decision makers who are responsible for deciding whether each of the proposals assigned to the committee should be accepted or rejected.

While the decision makers identify with the objectives of the organization, they differ in their ability to identify the state of the world that is relevant for each proposal. A decision maker's ability to identify the state of the world can be represented by the probability that he makes the correct assessment of a proposal. We assume that the organization employs decision makers with $s \geq 2$ different skill levels as defined by the probabilities that the decision makers assess a proposal correctly. Specifically, for $i=1,2, \ldots, s$, let $N_{i}$ be a positive and even number of decision makers who assess a proposal correctly with probability $p_{i}$, where $\sum_{i=1}^{s} N_{i}=2 N, \frac{1}{2}<p_{i}<1 \forall i$, and $p_{i} \neq p_{j} \forall i \neq j$. A decision maker's ability to make the correct assessment is the same in the two states. Furthermore, his assessment of a proposal is independent of his assessment of any other proposal and of the other decision makers' assessments.

Within a committee, each member expresses his true assessment by voting "yes" to a proposal that the member thinks should be adopted, and "no" to one he thinks should be rejected. Each committee's collective decision on each proposal is determined by a simple majority rule. Now, let $G\left(n_{1}, \ldots, n_{s}\right)$ denote the probability that the committee makes the correct decision by a simple majority rule, where $n_{i} \geq 0$ is the number of committee members with skill level $p_{i}$ and $\sum_{i=1}^{s} n_{i}=N$. The objective of the organization is to maximize the average probability of making correct decisions. Therefore, the organization chooses how many decision makers at each skill level to allocate to each committee in order to maximize 
$\frac{1}{2}\left[G\left(n_{1}, \ldots, n_{s}\right)+G\left(N_{1}-n_{1}, \ldots, N_{s}-n_{s}\right)\right]$.

\section{Symmetric Committees}

Let $\mathcal{N}$ denote a profile of $N$ decision makers. Also, let $\mathcal{N}_{i^{v} j^{w}}$ denote the profile of $N+v+w$ decision makers if, starting from $\mathcal{N}$, the number of decision makers with skill level $p_{i}$ is increased by $v$ and the number of decision makers with skill level $p_{j} \neq p_{i}$ is increased by $w$, where $v$ and $w$ are integers. Furthermore, let $\Gamma_{\uparrow}(M, \mathcal{N})$ denote the probability that at least $M$ decision makers in $\mathcal{N}$ make the correct assessment, and $\Gamma(M, \mathcal{N})$ denote that exactly $M$ make the correct assessment. Finally, for $w=-v$ let

$$
\Delta\left(\frac{N+1}{2}, \mathcal{N}_{i^{v} j^{-v}}\right) \equiv \Gamma_{\uparrow}\left(\frac{N+1}{2}, \mathcal{N}_{i^{v+1} j^{-v-1}}\right)-\Gamma_{\uparrow}\left(\frac{N+1}{2}, \mathcal{N}_{i^{v} j^{-v}}\right)
$$

denote the change in the probability that a simple majority makes the correct decision if, starting with a committee of decision makers with profile $\mathcal{N}_{i^{v} j^{-v}}$, one decision maker with skill level $p_{i}$ replaces one with skill level $p_{j}$. We now prove that the change in the probability decreases (increases) with the number of decision makers with the skill level $p_{i}\left(p_{j}\right)$ already in the committee:

Lemma 1: $\Delta\left[(N+1) / 2, \mathcal{N}_{i^{v} j^{-v}}\right]$ decreases in $v$.

Proof: The only case in which the exchange of a decision maker with skill level $p_{i}$ with a decision maker with skill level $p_{j}$ can make a difference in the committee's decision is if the exchanged decision maker happens to be pivotal; that is, if the rest of the committee members are divided evenly for and against the proposal. We therefore first derive an expression for $\Delta\left[(N+1) / 2, \mathcal{N}_{i^{v} j^{-v}}\right]$ in terms of the probability that the exchanged decision maker is pivotal.

The probability that in a committee with profile $\mathcal{N}_{i^{v+1} j^{-v-1}}$ of decision makers, at least 
$(N+1) / 2$ of these will make the correct assessment is given by

$$
\begin{aligned}
& \Gamma_{\uparrow}\left(\frac{N+1}{2}, \mathcal{N}_{i^{v+1} j^{-v-1}}\right) \\
= & p_{i} \Gamma_{\uparrow}\left(\frac{N-1}{2}, \mathcal{N}_{i^{v} j^{-v-1}}\right)+\left(1-p_{i}\right) \Gamma_{\uparrow}\left(\frac{N+1}{2}, \mathcal{N}_{i^{v} j^{-v-1}}\right) .
\end{aligned}
$$

Similarly, the probability that in a committee with profile $\mathcal{N}_{i^{v} j^{-v}}$ of decision makers, at least $(N+1) / 2$ of these will make the correct assessment is given by

$$
\begin{aligned}
& \Gamma_{\uparrow}\left(\frac{N+1}{2}, \mathcal{N}_{i^{v} j^{-v}}\right) \\
= & p_{j} \Gamma_{\uparrow}\left(\frac{N-1}{2}, \mathcal{N}_{i^{v} j^{-v-1}}\right)+\left(1-p_{j}\right) \Gamma_{\uparrow}\left(\frac{N+1}{2}, \mathcal{N}_{i^{v} j^{-v-1}}\right) .
\end{aligned}
$$

It follows that in a committee with profile $\mathcal{N}_{i^{v} j^{-v}}$ of decision makers, the change in the probability of making the correct decision due to the exchange of decision makers is

$$
\begin{aligned}
& \Delta\left(\frac{N+1}{2}, \mathcal{N}_{i^{v} j^{-v}}\right) \\
= & \left(p_{i}-p_{j}\right)\left[\Gamma_{\uparrow}\left(\frac{N-1}{2}, \mathcal{N}_{i^{v} j^{-v-1}}\right)-\Gamma_{\uparrow}\left(\frac{N+1}{2}, \mathcal{N}_{i^{v} j^{-v-1}}\right)\right] \\
= & \left(p_{i}-p_{j}\right) \Gamma\left(\frac{N-1}{2}, \mathcal{N}_{i^{v} j^{-v-1}}\right),
\end{aligned}
$$

where $p_{i}-p_{j}$ is the change in the probability that the exchanged decision maker makes the correct assessment and $\Gamma\left[(N-1) / 2, \mathcal{N}_{i^{v} j^{-v-1}}\right]$ is the probability that the exchanged decision maker is pivotal.

By substituting $v+1$ in place of $v$ in the above formula, we obtain that the change in the probability of making the correct decision due to the change of decision makers in a committee with profile $\mathcal{N}_{i^{v+1} j^{-v-1}}$ is

$$
\begin{aligned}
& \Delta\left(\frac{N+1}{2}, \mathcal{N}_{i^{v+1} j^{-v-1}}\right) \\
= & \left(p_{i}-p_{j}\right) \Gamma\left(\frac{N-1}{2}, \mathcal{N}_{i^{v+1} j^{-v-2}}\right) .
\end{aligned}
$$

Accordingly, the change in the probability of making the correct decision caused by exchanging the decision makers in a committee with profile $\mathcal{N}_{i^{v+1} j^{-v-1}}$ less the corresponding change 
from a similar exchange in a committee with profile $\mathcal{N}_{i^{v} j^{-v}}$ is

$$
\begin{aligned}
& \Delta\left(\frac{N+1}{2}, \mathcal{N}_{i^{v+1} j^{-v-1}}\right)-\Delta\left(\frac{N+1}{2}, \mathcal{N}_{i^{v} j^{-v}}\right) \\
= & \left(p_{i}-p_{j}\right)\left[\Gamma\left(\frac{N-1}{2}, \mathcal{N}_{i^{v+1} j^{-v-2}}\right)-\Gamma\left(\frac{N-1}{2}, \mathcal{N}_{i^{v} j^{-v-1}}\right)\right] .
\end{aligned}
$$

The term in the square brackets is the probability that the exchanged decision maker is pivotal in a committee where the other members have the profile $\mathcal{N}_{i^{v+1} j^{-v-2}}$, less the probability that the exchanged decision maker is pivotal in a committee with one less decision maker with skill level $p_{i}$ and one more with skill level $p_{j}$ among the other committee members. Since

$$
\begin{aligned}
& \Gamma\left(\frac{N-1}{2}, \mathcal{N}_{i^{v+1} j^{-v-2}}\right) \\
= & p_{i} \Gamma\left(\frac{N-3}{2}, \mathcal{N}_{i^{v} j^{-v-2}}\right)+\left(1-p_{i}\right) \Gamma\left(\frac{N-1}{2}, \mathcal{N}_{i^{v} j^{-v-2}}\right)
\end{aligned}
$$

and

$$
\begin{aligned}
& \Gamma\left(\frac{N-1}{2}, \mathcal{N}_{i^{v} j^{-v-1}}\right) \\
= & p_{j} \Gamma\left(\frac{N-3}{2}, \mathcal{N}_{i^{v} j^{-v-2}}\right)+\left(1-p_{j}\right) \Gamma\left(\frac{N-1}{2}, \mathcal{N}_{i^{v} j^{-v-2}}\right),
\end{aligned}
$$

we can write (1) as

$$
\left(p_{i}-p_{j}\right)^{2}\left[\Gamma\left(\frac{N-3}{2}, \mathcal{N}_{i^{v} j^{-v-2}}\right)-\Gamma\left(\frac{N-1}{2}, \mathcal{N}_{i^{v} j^{-v-2}}\right)\right],
$$

which is negative since $\Gamma\left[(N-3) / 2, \mathcal{N}_{i^{v} j^{-v-2}}\right]<\Gamma\left[(N-1) / 2, \mathcal{N}_{i^{v} j^{-v-2}}\right]$.

As a consequence, $\Delta\left[(N+1) / 2, \mathcal{N}_{i^{v} j^{-v}}\right]$ decreases in $v$; that is, the change in the probability of the committee making the correct decision when one decision maker with skill level $p_{i}$ replaces a decision maker with skill level $p_{j}$ (and all the other committee members are unchanged) is a decreasing function of the number of decision makers with skill level $p_{i}$ already in the committee.

The intuition of Lemma 1 is that the higher (lower) the skill levels of $N-1$ committee members, the less (more) likely it is that the $N$ th member is pivotal. Suppose that a 
decision maker with a higher skill level replaces a decision maker with a lower skill level. The change in the probability that a simple majority makes the correct decision is positive, and it increases at a decreasing rate with the number of committee members with the same higher skill level who are already in the committee. Suppose instead that a decision maker with a lower skill level replaces a decision maker with a higher skill level. Since adding a decision maker with a lower skill level is equivalent to subtracting a decision maker with a higher skill level, the change in the probability that a simple majority makes the correct decision is negative and its absolute value increases at a increasing rate with the number of committee members with the same lower skill level who are already in the committee.

We now establish that it is not optimal for an organization to divide all but two types of decision makers evenly among the committees. Let $\widehat{\mathcal{N}}=\left(N_{1} / 2, N_{2} / 2, \ldots, N_{s} / 2\right)$ denote the profile of the decision makers in a symmetric committee with $N$ members.

Theorem 1: Dividing the decision makers to profiles $\widehat{\mathcal{N}}_{i^{v} j^{-v}}$ and $\widehat{\mathcal{N}}_{i^{-v} j^{v}}, v \neq 0$, is not optimal.

Proof: Maximizing $\frac{1}{2}\left[G\left(\widehat{\mathcal{N}}_{i^{v} j^{-v}}\right)+G\left(\widehat{\mathcal{N}}_{i^{-v} j^{v}}\right)\right]$ with respect to $v$ is equivalent to maximizing

$$
\frac{1}{2}\left[\Gamma_{\uparrow}\left(\frac{N+1}{2}, \widehat{\mathcal{N}}_{i^{v} j^{-v}}\right)+\Gamma_{\uparrow}\left(\frac{N+1}{2}, \widehat{\mathcal{N}}_{i^{-v} j^{v}}\right)\right]
$$

with respect to $v$. If one decision maker with skill level $p_{i}$ is moved to the committee with $\widehat{\mathcal{N}}_{i^{v} j^{-v}}$ from the committee with $\widehat{\mathcal{N}}_{i^{-v} j^{v}}$ in lieu of a decision maker with skill level $p_{j}$ that is moved the other way, the average probability that the committees will make the correct decision changes by

$$
\begin{aligned}
& \frac{1}{2}\left[\Gamma_{\uparrow}\left(\frac{N+1}{2}, \widehat{\mathcal{N}}_{i^{v+1} j^{-v-1}}\right)+\Gamma_{\uparrow}\left(\frac{N+1}{2}, \widehat{\mathcal{N}}_{i^{-v-1} j^{v+1}}\right)\right] \\
& -\frac{1}{2}\left[\Gamma_{\uparrow}\left(\frac{N+1}{2}, \widehat{\mathcal{N}}_{i^{v} j^{-v}}\right)+\Gamma_{\uparrow}\left(\frac{N+1}{2}, \widehat{\mathcal{N}}_{i^{-v} j^{v}}\right)\right] \\
= & \frac{1}{2}\left[\Delta\left(\frac{N+1}{2}, \widehat{\mathcal{N}}_{i^{v} j^{-v}}\right)-\Delta\left(\frac{N+1}{2}, \widehat{\mathcal{N}}_{i^{-v-1} j^{v+1}}\right)\right] .
\end{aligned}
$$


Since Lemma 1 shows that $\Delta\left[(N+1) / 2, \widehat{\mathcal{N}}_{i^{v} j^{-v}}\right]$ decreases in $v$ and that $\Delta\left[(N+1) / 2, \widehat{\mathcal{N}}_{i^{-v-1} j^{v+1}}\right]$ increases in $v$, it follows that $(2)$ decreases in $v$. As Lemma 1 also implies that (2) for $v=-1$ equals

$$
\frac{1}{2}\left[\Delta\left(\frac{N+1}{2}, \widehat{\mathcal{N}}_{i^{-1} j^{1}}\right)-\Delta\left(\frac{N+1}{2}, \widehat{\mathcal{N}}\right)\right]>0,
$$

and for $v=0$ equals

$$
\frac{1}{2}\left[\Delta\left(\frac{N+1}{2}, \widehat{\mathcal{N}}\right)-\Delta\left(\frac{N+1}{2}, \widehat{\mathcal{N}}_{i^{-1} j^{1}}\right)\right]<0,
$$

it follows that the maximum value of $\frac{1}{2}\left[G\left(\widehat{\mathcal{N}}_{i^{v} j^{-v}}\right)+G\left(\widehat{\mathcal{N}}_{i^{-v} j^{v}}\right)\right]$ is obtained for only $v=0$.

Accordingly, starting with two symmetric committees, it is never optimal to reassign decision makers with two skill levels so that decision makers with one skill level in one committee are exchanged for the same number of decision makers with the other skill level in the other committee. Hence, if for some exogenous reason decision makers of only two skill levels have to be added to or removed from symmetric committees and the organization is not able to move any of the existing decision makers from one committee to the other, then the added or removed decision makers have to be divided evenly so that the changed committees are also symmetric.

Corollary 1: If $s=2$, symmetric committees are uniquely optimal.

Proof: If $s=2$, then $\widehat{\mathcal{N}}_{i^{v} j^{-v}}=\left(N_{1} / 2+v, N_{2} / 2-v\right)$ and $\widehat{\mathcal{N}}_{i^{-v} j^{v}}=\left(N_{1} / 2-v, N_{2} / 2+v\right)$ so that the profiles of the decision makers in the two committees depend only on $v$. Since Theorem 1 shows that $\widehat{\mathcal{N}}_{i^{v} j^{-v}}$ and $\widehat{\mathcal{N}}_{i^{-v} j^{v}}, v \neq 0$, is not optimal, it follows that $v=0$ is uniquely optimal.

Thus, if there are only two types of decision makers, i.e., high-skilled and low-skilled, it is optimal for the organization to allocate the two types evenly so that the compositions of 
the committees are symmetric. That is, regardless of the relative numbers of the decision makers with the two skill levels, each committee should have the same number of high- and low-skilled decision makers.

Suppose that decision makers can have only two skill levels. One implication of Corollary 1 is that if the decision makers are divided optimally among the two committees, and the organization is then faced with a need to change the size of the committees by either adding or removing the same number of decision makers from each committee, the new optimal allocation of decision members can be obtained without the organization having to move any decision maker from one committee to the other. In other words, suppose that the allocation of decision makers is sequential, i.e., the division of the decision makers among the two committees takes place in steps such that an even number of makers are first divided among the two committees, then an additional even number of decision makers are divided among the same two committees without moving any of the already allocated decision makers, etc. Corollary 1 then implies that this can be done such that, at each step, the average probability of making the correct decisions is maximized for the committee size at that step.

With two types of decision makers, one possible application of our model is to a court system. To illustrate, suppose that all cases are tried by a panel of judges and that a judge can be classified as either junior or senior, with the latter being higher skilled (due to more expertise and experience on the bench) than the former. A pertinent question would then be how to allocate judges to the different courts so as to maximize the number of correct decisions. Corollary 1 shows that it would be inefficient to have some panels consisting of only junior judges and others of only senior judges. Rather, it would be optimal that junior and senior judges be distributed evenly over the different courts.

Corollary 2: If $s=3$ and $N_{i}=2 \forall i$, symmetric committees are uniquely optimal.

Proof: If $s=3$, an asymmetric committee will have two decision makers of one type, one decision maker of another type, and no decision maker of the last type. Therefore, all 
the asymmetric committees can be obtained from symmetric committees by $v=1$. Since Theorem 1 shows that $v=1$ is not optimal, it follows that $v=0$ and hence that the symmetric committees are uniquely optimal.

The underlying logic is that, starting with an even distribution of decision makers, any composition of two three-member committees can be obtained by a single reassignment of one decision maker from one committee to the other. The reason is that the reassignment of two members from each committee to the other is equivalent, in terms of the composition of the committees, to the reassignment of one member from each committee to the other. ${ }^{7}$

\section{Asymmetric Committees}

If there are more than two skill levels and more than three members in each committee, then the committees should generally not be symmetric. Indeed, Theorem 2 will establish that starting with an even division of decision makers among the two committees, a reassignment that involves decision makers with more than two skill levels may increase the average probability that the committees make the right decisions. In that case, symmetric committees are not optimal. The reason is that, in contrast to the assumption of Lemma 1, starting from an even distribution of decision makers among the committees, it is necessary to exchange decision makers with more than two skill levels between the committees in order to obtain is

${ }^{7}$ Let $p_{i}, p_{j}$, and $p_{k}$ denote the three skill levels. The average probability of making the correct decisions

$$
p_{i} p_{j}+p_{i} p_{k}+p_{j} p_{k}-2 p_{i} p_{j} p_{k}
$$

if the committees are symmetric, and

$$
\frac{1}{2}\left(p_{j}^{2}+2 p_{i} p_{j}-2 p_{i} p_{j}^{2}+p_{k}^{2}+2 p_{i} p_{k}-2 p_{i} p_{k}^{2}\right)
$$

if the committees are asymmetric. Hence, the former exceeds the latter by

$$
\begin{aligned}
& p_{i} p_{j}+p_{i} p_{k}+p_{j} p_{k}-2 p_{i} p_{j} p_{k}-\frac{1}{2}\left(p_{j}^{2}+2 p_{i} p_{j}-2 p_{i} p_{j}^{2}+p_{k}^{2}+2 p_{i} p_{k}-2 p_{i} p_{k}^{2}\right) \\
= & \left(p_{i}-\frac{1}{2}\right)\left(p_{j}-p_{k}\right)^{2} \\
> & 0 .
\end{aligned}
$$


some of the possible allocations of decision makers with different skill levels to the committees. In general, such an exchange does not cause a decrease in the average probability that the committees will make the correct decision.

To prove that asymmetric committees may be optimal, we examine cases in which, starting with symmetric committees, two decision makers in one committee are exchanged with two decision makers in the other committee. ${ }^{8}$ For simplicity, therefore, assume that there are five different skill levels with two decision makers at each of the four skill levels and two or more decision makers at the fifth skill level. Let the four skill levels with two decision makers in each be denoted by $p_{i}, p_{j}, p_{k}$, and $p_{\ell}$, and the fifth skill level by $p$. In this case $\widehat{\mathcal{N}}$ denotes the profile of decision makers with $n_{i}=n_{j}=n_{k}=n_{\ell}=1$ and $N-4$ decision makers with skill level $p$. Further, let $\widehat{\mathcal{N}}_{i^{v} j^{w} k^{x} \ell^{y}}$ denote the profile of $N+v+w+x+y$ decision makers if, starting from $\widehat{\mathcal{N}}$, the number of decision makers with skill level $p_{i}, p_{j}, p_{k}$, and $p_{\ell}$ are increased by $v, w, x$, and $y$, respectively, where $v, w, x$, and $y$ are integers. Finally, let $a \equiv p_{k} p_{\ell}-p_{i} p_{j}$ be the probability that a pair of decision makers with skill levels $p_{k}$ and $p_{\ell}$ both make the correct assessment less the probability that a pair of decision makers with skill levels $p_{i}$ and $p_{j}$ both make the correct assessment. Also, let $b \equiv p_{k}+p_{\ell}-p_{i}-p_{j}$ so that $b-a$ is the probability that at least one of the decision makers in a pair with skill levels $p_{k}$ and $p_{\ell}$ makes the correct assessment less the probability that at least one of the decision makers in a pair with skill levels $p_{i}$ and $p_{j}$ makes the correct assessment. Note that if $a=0 \quad(b=0)$, the skill levels of the two pairs of decision makers have the same geometric (arithmetic) means. Furthermore, $a=0 \Rightarrow b \neq 0$ and $b=0 \Rightarrow a \neq 0 .{ }^{9}$

\footnotetext{
8 All possible compositions of the committees can be obtained from symmetric committees by reassigning at most $(N-1) / 2$ decision makers from one committee to the other (as already explained for the case of $N=3$ ). In the case of $N=5$, therefore, it suffices to consider at most two reassignments.

9 Since $a=0$ implies that

$$
\begin{aligned}
b p_{i} & =p_{i} p_{k}+p_{i} p_{\ell}-p_{i}^{2}-p_{k} p_{\ell} \\
& =\left(p_{i}-p_{\ell}\right)\left(p_{k}-p_{i}\right),
\end{aligned}
$$

it follows that $a=b=0$ is infeasible because it would require that $p_{i}=p_{\ell}$ and $p_{j}=p_{k}$ or that $p_{i}=p_{k}$ and $p_{j}=p_{\ell}$.
} 
The average probability of making the correct decision with asymmetric committees of decision makers having profiles $\widehat{\mathcal{N}}_{i^{-1} j^{-1} k^{1} \ell^{1}}$ and $\widehat{\mathcal{N}}_{i^{1} j^{1} k^{-1} \ell^{-1}}$ less the average probability of making the correct decision with symmetric committees is

$$
\begin{aligned}
& \frac{1}{2}\left[\Gamma_{\uparrow}\left(\frac{N+1}{2}, \widehat{\mathcal{N}}_{i^{-1} j^{-1} k^{1} \ell^{1}}\right)+\Gamma_{\uparrow}\left(\frac{N+1}{2}, \widehat{\mathcal{N}}_{i^{1} j^{1} k^{-1} \ell^{-1}}\right)\right]-\Gamma_{\uparrow}\left(\frac{N+1}{2}, \widehat{\mathcal{N}}\right) \\
= & \frac{1}{2}\left\{p_{k} p_{\ell} \Gamma\left(\frac{N-3}{2}, \widehat{\mathcal{N}}_{i^{-1} j^{-1}}\right)+\left[1-\left(1-p_{k}\right)\left(1-p_{\ell}\right)\right] \Gamma\left(\frac{N-1}{2}, \widehat{\mathcal{N}}_{i^{-1} j^{-1}}\right)\right. \\
& +\Gamma_{\uparrow}\left(\frac{N+1}{2}, \widehat{\mathcal{N}}_{i^{-1} j^{-1}}\right)+p_{i} p_{j} \Gamma\left(\frac{N-3}{2}, \widehat{\mathcal{N}}_{k^{-1} \ell^{-1}}\right) \\
& \left.+\left[1-\left(1-p_{i}\right)\left(1-p_{j}\right)\right] \Gamma\left(\frac{N-1}{2}, \widehat{\mathcal{N}}_{k^{-1} \ell^{-1}}\right)+\Gamma_{\uparrow}\left(\frac{N+1}{2}, \widehat{\mathcal{N}}_{k^{-1} \ell^{-1}}\right)\right\} \\
& -\frac{1}{2}\left\{p_{k} p_{\ell} \Gamma\left(\frac{N-3}{2}, \widehat{\mathcal{N}}_{k^{-1} \ell^{-1}}\right)+\left[1-\left(1-p_{k}\right)\left(1-p_{\ell}\right)\right] \Gamma\left(\frac{N-1}{2}, \widehat{\mathcal{N}}_{k^{-1} \ell^{-1}}\right)\right. \\
& +\Gamma_{\uparrow}\left(\frac{N+1}{2}, \widehat{\mathcal{N}}_{k^{-1} \ell^{-1}}\right)+p_{i} p_{j} \Gamma\left(\frac{N-3}{2}, \widehat{\mathcal{N}}_{i^{-1} j^{-1}}\right) \\
& \left.+\left[1-\left(1-p_{i}\right)\left(1-p_{j}\right)\right] \Gamma\left(\frac{N-1}{2}, \widehat{\mathcal{N}}_{i^{-1} j^{-1}}\right)+\Gamma_{\uparrow}\left(\frac{N+1}{2}, \widehat{\mathcal{N}}_{i^{-1} j^{-1}}\right)\right\} .
\end{aligned}
$$

Using $a$ and $b$ this becomes

$$
\begin{aligned}
& \frac{1}{2}\left[a \Gamma\left(\frac{N-3}{2}, \widehat{\mathcal{N}}_{i^{-1} j^{-1}}\right)+(b-a) \Gamma\left(\frac{N-1}{2}, \widehat{\mathcal{N}}_{i^{-1} j^{-1}}\right)\right] \\
& -\frac{1}{2}\left[a \Gamma\left(\frac{N-3}{2}, \widehat{\mathcal{N}}_{k^{-1} \ell^{-1}}\right)+(b-a) \Gamma\left(\frac{N-1}{2}, \widehat{\mathcal{N}}_{k^{-1} \ell^{-1}}\right)\right] \\
= & \frac{1}{2} a\left[\Gamma\left(\frac{N-3}{2}, \widehat{\mathcal{N}}_{i^{-1} j^{-1}}\right)-\Gamma\left(\frac{N-3}{2}, \widehat{\mathcal{N}}_{k^{-1} \ell^{-1}}\right)\right] \\
& +\frac{1}{2}(b-a)\left[\Gamma\left(\frac{N-1}{2}, \widehat{\mathcal{N}}^{i^{-1} j^{-1}}\right)-\Gamma\left(\frac{N-1}{2}, \widehat{\mathcal{N}}_{k^{-1} \ell^{-1}}\right)\right] .
\end{aligned}
$$

The expression in the first square brackets is the difference between the probability that there are exactly $(N-3) / 2$ correct assessments being made by decision makers with profile $\mathcal{N}_{i^{-1} j^{-1}}$ and with profile $\mathcal{N}_{k^{-1} \ell^{-1}}$. Therefore, the first expression in (3) is the change in the average probability that the committees make the correct decisions if the exchanged decision makers both make the correct assessment in order for the committee to decide correctly. 
The expression in the second square brackets in (3) is the difference between the probability that there are exactly $(N-1) / 2$ correct assessments being made by decision makers with profile $\widehat{\mathcal{N}}_{i^{-1} j^{-1}}$ and with profile $\widehat{\mathcal{N}}_{k^{-1} \ell^{-1}}$. Accordingly, the second expression in (3) is the change in the average probability that the committees make the correct decision if at least one of the exchanged decision makers make the correct assessment in order for the committee to decide correctly.

Consider now the case when $N=5$. Suppose that the products of the exchanged decision makers' skill levels are the same so that $a=p_{k} p_{\ell}-p_{i} p_{j}=0$. The difference in the probability that at least one correct assessment is being made by the exchanged decision makers is then equal to the difference in the sums of the exchanged decision makers' skill levels, i.e., $b=p_{k}+p_{\ell}-p_{i}-p_{j}$. At the same time, the difference in the probability that two correct assessments are being made by the three decision makers remaining in the committees is equal to the difference in the sums of the exchanged decision makers' skill levels times the fifth decision maker's skill level, i.e., $b p .^{10}$ Accordingly, the difference between the average probability of making the correct decision with asymmetric and symmetric committees (i.e., expression (3)) is $\frac{1}{2}$ times the product of $b$ and $b p$, and, since $b^{2}>0$, it is positive. That is, if $a=0$, then a symmetric committee is not optimal.

We now provide more general conditions under which symmetric committees are not optimal:

Theorem 2: If $a b \leq 0$ and $p<\frac{1}{2}+1 /(N-3)$, optimal committees are asymmetric.

Proof: Let $\overline{\mathcal{N}} \equiv \widehat{\mathcal{N}}_{i^{-1} j^{-1} k^{-1} \ell^{-1}}$. The Appendix shows that (3) can be written as

$$
\begin{aligned}
& { }^{10} \text { If } a=0 \text {, then } \\
& \qquad \begin{aligned}
& {\left[\Gamma\left(2, \widehat{\mathcal{N}}_{i^{-1} j^{-1}}\right)-\Gamma\left(2, \widehat{\mathcal{N}}_{k^{-1} \ell^{-1}}\right)\right] } \\
= & p_{k} p_{\ell}(1-p)+p_{k}\left(1-p_{\ell}\right) p+\left(1-p_{k}\right) p_{\ell} p-p_{i} p_{j}(1-p)-p_{i}\left(1-p_{j}\right) p-\left(1-p_{i}\right) p_{j} p \\
= & b p .
\end{aligned}
\end{aligned}
$$




$$
\begin{aligned}
& \frac{1}{2}\left[a^{2} \Gamma\left(\frac{N-7}{2}, \overline{\mathcal{N}}\right)+a(2 b-3 a) \Gamma\left(\frac{N-5}{2}, \overline{\mathcal{N}}\right)\right. \\
& \left.+\left(3 a^{2}+b^{2}-4 a b\right) \Gamma\left(\frac{N-3}{2}, \overline{\mathcal{N}}\right)-(b-a)^{2} \Gamma\left(\frac{N-1}{2}, \overline{\mathcal{N}}\right)\right] .
\end{aligned}
$$

First, suppose that $N=5$. Then $p<\frac{1}{2}+1 /(N-3)$ is always satisfied. Since $\Gamma[(N-7) / 2, \overline{\mathcal{N}}]=0 ; \Gamma[(N-5) / 2, \overline{\mathcal{N}}]=1-p ; \Gamma[(N-3) / 2, \overline{\mathcal{N}}]=p ;$ and $\Gamma[(N-1) / 2, \overline{\mathcal{N}}]=$ 0 , it follows that (4) is equal to

$$
\begin{aligned}
& \frac{1}{2}\left[a(2 b-3 a)(1-p)+\left(3 a^{2}+b^{2}-4 a b\right) p\right] \\
= & \frac{1}{2}\left[\left(3 a^{2}-2 a b\right)(2 p-1)+\left(b^{2}-2 a b\right) p\right] .
\end{aligned}
$$

Thus, if $a=0$, then (4) is equal to $\frac{1}{2} b^{2} p>0$, while if $b=0$, then (4) is equal to $3 a^{2}\left(p-\frac{1}{2}\right)>0$. Furthermore, if $a b<0$, then $3 a^{2}-2 a b>0$ and $b^{2}-2 a b>0$ so that (4) is positive. This completes the proof for $N=5$.

Next, suppose that $N \geq 7$. If $a=0$, then (4) equals

$$
\begin{aligned}
& \frac{1}{2} b^{2}\left[\Gamma\left(\frac{N-3}{2}, \overline{\mathcal{N}}\right)-\Gamma\left(\frac{N-1}{2}, \overline{\mathcal{N}}\right)\right] \\
= & \frac{1}{2} b^{2}\left[\left(\begin{array}{c}
N-4 \\
\frac{N-3}{2}
\end{array}\right) p^{(N-3) / 2}(1-p)^{(N-5) / 2}-\left(\begin{array}{c}
N-4 \\
\frac{N-1}{2}
\end{array}\right) p^{(N-1) / 2}(1-p)^{(N-7) / 2}\right] \\
= & \frac{b^{2}(N-4) ! p^{(N-3) / 2}(1-p)^{(N-7) / 2}[(N-1) / 2-p(N-3)]}{2\left(\frac{N-1}{2}\right) !\left(\frac{N-5}{2}\right) !}
\end{aligned}
$$

which is positive since $p<\frac{1}{2}+1 /(N-3)$.

If $b=0$, then (4) equals

$$
\frac{1}{2} a^{2}\left[\Gamma\left(\frac{N-7}{2}, \overline{\mathcal{N}}\right)-3 \Gamma\left(\frac{N-5}{2}, \overline{\mathcal{N}}\right)+3 \Gamma\left(\frac{N-3}{2}, \overline{\mathcal{N}}\right)-\Gamma\left(\frac{N-1}{2}, \overline{\mathcal{N}}\right)\right]
$$




$$
\begin{aligned}
= & \frac{1}{2} a^{2}\left[\left(\begin{array}{c}
N-4 \\
\frac{N-7}{2}
\end{array}\right) p^{(N-7) / 2}(1-p)^{(N-1) / 2}-3\left(\begin{array}{c}
N-4 \\
\frac{N-5}{2}
\end{array}\right) p^{(N-5) / 2}(1-p)^{(N-3) / 2}\right. \\
& \left.+3\left(\begin{array}{c}
N-4 \\
\frac{N-3}{2}
\end{array}\right) p^{(N-3) / 2}(1-p)^{(N-5) / 2}-\left(\begin{array}{c}
N-4 \\
\frac{N-1}{2}
\end{array}\right) p^{(N-1) / 2}(1-p)^{(N-7) / 2}\right] \\
= & \frac{4 a^{2}(N-2)(N-4) ! p^{(N-7) / 2}(1-p)^{(N-7) / 2}(2 p-1)}{\left(\frac{N-1}{2}\right) !\left(\frac{N-5}{2}\right) !}\left[\frac{3}{4(N-2)}-\left(p-\frac{1}{2}\right)^{2}\right],
\end{aligned}
$$

which is positive since $p<\frac{1}{2}+1 /(N-3) \Rightarrow\left(p-\frac{1}{2}\right)^{2}<3 /[4(N-2)]$.

Finally, if $a b<0$, we write $(4)$ as

$$
\begin{aligned}
& \frac{1}{2}\left(a^{2}\left\{3\left[\Gamma\left(\frac{N-3}{2}, \overline{\mathcal{N}}\right)-\Gamma\left(\frac{N-5}{2}, \overline{\mathcal{N}}\right)\right]+\left[\Gamma\left(\frac{N-7}{2}, \overline{\mathcal{N}}\right)-\Gamma\left(\frac{N-1}{2}, \overline{\mathcal{N}}\right)\right]\right\}\right. \\
& -2 a b\left[\Gamma\left(\frac{N-3}{2}, \overline{\mathcal{N}}\right)-\Gamma\left(\frac{N-5}{2}, \overline{\mathcal{N}}\right)\right] \\
& \left.+\left(b^{2}-2 a b\right)\left[\Gamma\left(\frac{N-3}{2}, \overline{\mathcal{N}}\right)-\Gamma\left(\frac{N-1}{2}, \overline{\mathcal{N}}\right)\right]\right) .
\end{aligned}
$$

Now,

$$
\begin{aligned}
& 3\left[\Gamma\left(\frac{N-3}{2}, \overline{\mathcal{N}}\right)-\Gamma\left(\frac{N-5}{2}, \overline{\mathcal{N}}\right)\right]+\left[\Gamma\left(\frac{N-7}{2}, \overline{\mathcal{N}}\right)-\Gamma\left(\frac{N-1}{2}, \overline{\mathcal{N}}\right)\right] \\
= & \frac{(N-4) !}{\left(\frac{N-7}{2}\right) !\left(\frac{N-7}{2}\right) !} p^{(N-7) / 2}(1-p)^{(N-7) / 2} \\
& \left\{\frac{3\left[p^{2}(1-p)-p(1-p)^{2}\right]}{\left.\left(\frac{N-5}{2}\right)^{2} \frac{N-3}{2}+\frac{(1-p)^{3}-p^{3}}{N-5} \frac{N-3}{2} \frac{N-1}{2}\right\}}\right. \\
= & \frac{4(N-2)(N-4) ! p^{(N-7) / 2}(1-p)^{(N-7) / 2}}{2\left(\frac{N-1}{2}\right) !\left(\frac{N-5}{2}\right) !}\left[\frac{3}{4(N-2)}-\left(p-\frac{1}{2}\right)^{2}\right],
\end{aligned}
$$

which has the same sign as $3 /[4(N-2)]-\left(p-\frac{1}{2}\right)^{2}$ and is therefore positive. Furthermore, $\Gamma[(N-3) / 2, \overline{\mathcal{N}}]-\Gamma[(N-5) / 2, \overline{\mathcal{N}}]>0$, and we have proven above that $\Gamma[(N-3) / 2, \overline{\mathcal{N}}]-$ $\Gamma[(N-1) / 2, \overline{\mathcal{N}}]>0$. Since $a b<0 \Rightarrow a^{2},-2 a b, b^{2}-2 a b>0$, it follows that (4) is positive. This completes the proof for $N \geq 7$. 
Even though our model assumes symmetry between the committees (they have the same size and make the same number of independent decisions) and between the alternatives (the prior probability and a decision maker's ability are independent of the state, and the net benefit of a correct decision is identical for each proposal and state), it is not always optimal to divide the decision makers into symmetric committees. This is due to the interaction of decision makers with more than two skill levels and does not occur when only two skill levels are being exchanged (cf. Lemma 1). Thus, while a decision maker with a higher skill level is always preferred to one with a lower skill, whether one pair of decision makers is preferred to another having one more skilled and one less skilled decision maker, depends not only on the skill levels of the two pairs of decision makers but also on the skill levels of the other decision makers in the committee. In particular, if pairs of decision makers are exchanged between symmetric committees, and the skill levels of the exchanged pair of decision makers have the same geometric or arithmetic mean, i.e., if $a=0$ or $b=0$, then asymmetric committees are always optimal for $N=5$ and, depending on the skill levels of the other decision makers, may be optimal for $N \geq 7 .^{11}$

A practical implication of Theorem 2 is that it may not be possible to allocate the decision makers sequentially such that, at each step, the average probability of making the correct decisions is maximized for the committee size at that step. To illustrate, if the organization starts out with two thee-member committees and two decision makers at each of three skill levels, then Corollary 2 shows that one decision maker at each skill level should be allocated to each of the committees. If, however, the organization subsequently wishes to switch to five-member committees and hires four additional decision makers, with two decision makers at each of two different skill levels, it might then not be desirable to add one of each type of the new decision makers to each committee. Rather, to obtain an optimal allocation of decision makers, the organization might have to add all the new decision makers to one

\footnotetext{
${ }^{11}$ If $a=0$, the exchange of decision makers also leaves the geometric means of the committee members' skill level unchanged; if $b=0$, the exchange of the decision makers also leaves the arithmetic means of the committee members' skill level unchanged.
} 
committee and switch two existing decision makers from that committee to the other. The upshot is that an organization that foresees a change in the size of the committees, but has no flexibility to switch decision makers between the committees, might be forced to choose between an optimal allocation of decision makers with the current size of the committees and an optimal allocation of decision makers with the future size of the committees.

\section{Conclusion}

Despite the obvious practical importance of determining how a given group of decision makers with different skill levels should be assigned to different committees, this problem has not previously been theoretically addressed. To fill this gap in the literature, we have examined the symmetric case of two independent committees of equal size that work in parallel and have to decide on an equal number of independent proposals using a simple majority rule. For each proposal, it is a priori equally likely that "acceptance" and "rejection" is the correct decision, and the net benefit from a correct decision is the same for all proposals.

We find that, in general, it is not optimal to strive for a symmetric composition of committees. That is, it may not be desirable to divide decision makers with different skill levels evenly among committees. However, if decision makers have only two skill levels, symmetric committees are optimal. 


\section{Appendix}

Since

$$
\begin{aligned}
& \Gamma\left(\frac{N-3}{2}, \widehat{\mathcal{N}}_{i^{-1} j^{-1}}\right) \\
= & p_{k} p_{\ell} \Gamma\left(\frac{N-7}{2}, \overline{\mathcal{N}}\right)+\left[p_{k}\left(1-p_{\ell}\right)+p_{\ell}\left(1-p_{k}\right)\right] \Gamma\left(\frac{N-5}{2}, \overline{\mathcal{N}}\right) \\
& +\left(1-p_{k}\right)\left(1-p_{\ell}\right) \Gamma\left(\frac{N-3}{2}, \overline{\mathcal{N}}\right)
\end{aligned}
$$

and

$$
\begin{aligned}
& \Gamma\left(\frac{N-3}{2}, \widehat{\mathcal{N}}_{k^{-1} \ell^{-1}}\right) \\
= & p_{i} p_{j} \Gamma\left(\frac{N-7}{2}, \overline{\mathcal{N}}\right)+\left[p_{i}\left(1-p_{j}\right)+p_{j}\left(1-p_{i}\right)\right] \Gamma\left(\frac{N-5}{2}, \overline{\mathcal{N}}\right) \\
& +\left(1-p_{j}\right)\left(1-p_{i}\right) \Gamma\left(\frac{N-3}{2}, \overline{\mathcal{N}}\right),
\end{aligned}
$$

it follows that

$$
\begin{aligned}
& \Gamma\left(\frac{N-3}{2}, \widehat{\mathcal{N}}_{i^{-1} j^{-1}}\right)-\Gamma\left(\frac{N-3}{2}, \widehat{\mathcal{N}}_{k^{-1} \ell^{-1}}\right) \\
= & a \Gamma\left(\frac{N-7}{2}, \overline{\mathcal{N}}\right)+(b-2 a) \Gamma\left(\frac{N-5}{2}, \overline{\mathcal{N}}\right)+(a-b) \Gamma\left(\frac{N-3}{2}, \overline{\mathcal{N}}\right) .
\end{aligned}
$$

Similarly,

$$
\begin{aligned}
& \Gamma\left(\frac{N-1}{2}, \widehat{\mathcal{N}}_{i^{-1} j^{-1}}\right)-\Gamma\left(\frac{N-1}{2}, \widehat{\mathcal{N}}_{k^{-1} \ell^{-1}}\right) \\
= & a \Gamma\left(\frac{N-5}{2}, \overline{\mathcal{N}}\right)+(b-2 a) \Gamma\left(\frac{N-3}{2}, \overline{\mathcal{N}}\right)+(a-b) \Gamma\left(\frac{N-1}{2}, \overline{\mathcal{N}}\right) .
\end{aligned}
$$

Consequently, (3) becomes

$$
\begin{aligned}
& \frac{1}{2}\left[a^{2} \Gamma\left(\frac{N-7}{2}, \overline{\mathcal{N}}\right)+a(2 b-3 a) \Gamma\left(\frac{N-5}{2}, \overline{\mathcal{N}}\right)\right. \\
& \left.+\left(3 a^{2}+b^{2}-4 a b\right) \Gamma\left(\frac{N-3}{2}, \overline{\mathcal{N}}\right)-(b-a)^{2} \Gamma\left(\frac{N-1}{2}, \overline{\mathcal{N}}\right)\right] .
\end{aligned}
$$




\section{References}

Aristotle, 350 BCE. Politics, part XVI. Translated by Benjamin Jowett.

Austen-Smith, D., Banks, J.S., 1996. Information aggregation, rationality, and the Condorcet jury theorem. American Political Science Review 90, 34-45.

Baharad, E., Nitzan, S., 2007. Scoring rules: An alternative parameterization. Economic Theory 30, 187-90.

Ben-Yashar, R., Kraus, S., 2002. Optimal collective dichotomous choice under quota constraints. Economic Theory 19, 839-852.

Ben-Yashar R., Milchtaich, I., 2007. First and second best voting rules in committees. Social Choice and Welfare 29, 453-86.

Ben-Yashar, R., Nitzan, S., 1997. The optimal decision rule for fixed-size committees in dichotomous choice situations: The general result. International Economic Review 38, $175-186$.

Börgers, T., 2004. Costly voting. American Economic Review 94, 57-66.

Campbell, C., 1999. Large electorates and decisive minorities. Journal of Political Economy 107, 1199-1217.

Condorcet, N.C., 1785: Essai sur l'application de l'analyse à la probabilitè des dècisions rendues à la pluralitè des voix, Paris. In: I. McLean and F. Hewitt, translators, 1994.

Dewatripont, M., Tirole, J., 1999. Advocates. Journal of Political Economy 107, 1-39.

Feddersen, T., Pesendorfer, W., 1998. Convicting the innocent: The inferiority of unanimous jury verdicts. American Political Science Review 92, 23-35.

Grofman, B., Feld, S.L., 1988. Rousseau's general will: A Condorcet perspective. American Political Science Review 82, 567-576.

Grofman, B., Owen, G., Feld, S.L., 1983. Thirteen theorems in search of the truth. Theory and Decision 15, 261-278.

Heywood, J., 1546. A dialogue conteinyng the nomber in effect of all the prouerbes in the Englishe tongue. 
Ladha, K.K., 1995. Information pooling through majority-rule voting: Condorcet's jury theorem with correlated votes. Journal of Economic Behavior and Organization 26, $353-372$.

Nitzan, S., Paroush, J., 1982. Optimal decision rules in uncertain dichotomous choice situations. International Economic Review 23, 289-297.

Palfrey, T.R., Rosenthal, H., 1983. A strategic calculus of voting. Public Choice 41, 7-53.

Palfrey, T.R., Rosenthal, H., 1985. Voter participation and strategic uncertainty. American Political Science Review 79, 62-78.

Sah, R.K., 1991. Fallibility in human organizations and political systems. Journal of Economic Perspectives 5, 67-88.

Sah, R.K., Stiglitz, J.E., 1985. The theory of economic organizations, human fallibility and economic organizations. American Economic Review 75, 292-97.

Shapley, L., Grofman, B., 1984. Optimizing group judgmental accuracy in the presence of interdependencies. Public Choice 43, 329-343.

Young, H.P., 1988. Condorcet's rule. American Political Science Review 82, 1231-1244. 